${ }^{2}$ Meyer et al. Progressive change in cerebral blood flow during the first three weeks after subarachnoid haemorrhage. Neurosurgery 1983;12:58-76.

\section{Maurice-Williams replies:}

I have carefully re-read the article concerned and I cannot see that I have misquoted it in my previous comment on the letter of Mr Scharr and Mr Neil-Dwyer. The subsequently published article does indeed report that administration of tranexamic acid appears to be associated with a fall in cerebral blood flow during the second week after subarachnoid haemorrhage. If this is confirmed it will clearly have very important implications with regard to the effect of anti-fibrinolytic drugs on patients with ruptured aneurysms. However it does not resolve the main point that I had hoped to get across in the paper that is the subject of this discussionnamely the differing time courses of confirmed re-bleeds and episodes of nonhaemorrhagic deterioration and the fact that the time course of the latter coincides with the time course of rebleeding as reported in earlier studies, suggesting that those studies may have confused these two events and thus significantly overestimated the incidence of early rebleeding.

\section{Notices}

The Commonwealth Association for Mental Handicap and Developmental Disabilities (CAMHDD) has recently been formed with the support of a launching grant from the Commonwealth Foundation and has as its principal aims the prevention and amelioration of mental handicap and related developmental disabilities in developing Commonwealth countries. It is hoped that its membership ultimately will be that of individual professional and non-professional workers either working within developing countries in the field of mental handicap or those in developed countries that have a particular interest in this field in such countries. It is hoped to establish ultimately a form of directory of interested workers and from time to time practically orientated workshops will be held in differing developing countries. Further details may be obtained from the Association's UK Representative: Dr Gwilym Hosking, Consultant Paediatric Neurologist, The Ryegate Centre, Children's Hospital, Sheffield S10 5DD, UK.

Symposium on Brain Metabolism. The Eleventh International Symposium of the Fulton Society on Brain Metabolism will be held on 2 October, 1983 in New Orleans, USA associated with the 108th Annual Meeting of the American Neurological Association. Details may be obtained from Professor Dr Victor Soriano, Calle Buenos Aires 363, Montevideo, Uruguay.

\section{Correction}

Motor Neuron Disease in England and Wales, 1959-1979 (J Neurol Neurosurg Psychiatry 1983;46:197-205)

The authors wish to correct the figures in the last column of table 1 , where the values for "Neurologists $/ 100,000$ population" were too high by a factor of 100 . Thus, for example, the figure for London should have been $1 \cdot 15$, for Greater Manchester 0.30 . 